\title{
Compreendendo a dinâmica assistencial do serviço de emergência psiquiátrica utilizando a avaliação de quarta geração*
}

\author{
Aline Aparecida Buriola ${ }^{1}$, Sonia Silva Marcon ${ }^{2}$, Luciane Prado Kantorski ${ }^{3}$, Laura Misue Matsuda ${ }^{4}$
}

\author{
* Resultado da Tese de Doutorado \\ intitulada "Avaliação de um serviço de \\ emergência psiquiátrica inserido em um \\ hospital geral por meio do método de \\ quarta geração", apresentada ao Programa \\ de Pós-Graduação em Enfermagem da \\ Universidade Estadual de Maringá, em \\ 2014. \\ ${ }^{1}$ Enfermeira, Doutora em Enfermagem. \\ Docente da Universidade do Oeste \\ Paulista. Presidente Prudente, SP, Brasil. E- \\ mail: aliburiola@bol.com.br. \\ ${ }^{2}$ Enfermeira, Doutora em Filosofia da \\ Enfermagem. Professor Titular da \\ Universidade Estadual de Maringá. \\ Maringá, PR, Brasil. E-mail: \\ soniasilva.marcon@gmail.com. \\ ${ }^{3}$ Enfermeira, Doutora em Enfermagem. \\ Professora Associada da Universidade \\ Federal de Pelotas. Pelotas, RS, Brasil. E- \\ mail: kantorski@uol.com.br. \\ ${ }^{4}$ Enfermeira, Doutora em Enfermagem. \\ Professora Adjunta da Universidade \\ Estadual de Maringá. Maringá, PR, Brasil. E- \\ mail: lauramisuem@gmail.com.
}

Recebido: 29/09/2016.

Aceito: 10/10/2017.

Publicado: 31/12/2017.

\section{RESUMO}

Estudo que objetivou compreender as reivindicações, preocupações e questões construídas por profissionais acerca da dinâmica assistencial de um serviço de emergência psiquiátrica. Para tanto, foi realizado um estudo de caso, por meio da avaliação de Quarta Geração, com 15 participantes. A coleta de dados ocorreu por meio de análise documental, entrevista semiestruturada e observação, e a análise pelo método comparativo constante. Assim emergiram dois eixos temáticos: a) Compreendendo a dinâmica assistencial do serviço de emergência Psiquiátrica e; b) A desarticulação da rede de atenção psicossocial como entrave para a satisfação sobre a assistência na emergência psiquiátrica. Considera-se que a dinâmica assistencial na Emergência Psiquiátrica extrapola o mero caráter de unidade de estabilização de pacientes com quadros de agudização do transtorno mental, uma vez que esta direciona o fluxo de usuários para o tratamento adequado na rede de atenção psicossocial.

Descritores: Serviços de Emergência Psiquiátrica; Assistência à Saúde Mental; Enfermagem Psiquiátrica; Avaliação em Saúde.

\section{INTRODUÇÃO}

A Política Nacional de Saúde Mental regulamenta a atenção em saúde mental no Brasil, por meio da Lei 10.216/2001, a qual abarca questões de cuidado psiquiátrico humanizado e resolutivo, utilizando como pilares para construção de seus pressupostos, o movimento de Reforma Psiquiátrica, construída na década de 1970, por Franco

Basaglia na Itália e, no mesmo período no Brasil, pelo movimento de trabalhadores da saúde mental e movimentos sociais ${ }^{(1-2)}$. 
A Reforma Psiquiátrica objetiva a implantação e a consolidação de uma Rede de Atenção Psicossocial, substitutiva à internação manicomial e asilar, composta por Centros de Atenção Psicossocial (CAPS); Centros de Atenção Psicossocial para álcool e drogas (CAPSad), Centros de Atenção Psicossocial Infantil (CAPSi), Ambulatórios de Saúde Mental; Centros de Convivência; Hospital Dia; Serviços de Emergência Psiquiátrica em Hospital Geral e; Leitos de Atenção Psiquiátrica, tidos como unidades de cuidado, inseridos em hospitais gerais $^{(3)}$.

As unidades de Emergência Psiquiátrica padecem para instaurar o cuidado articulado com a Rede Psicossocial, expondo a pessoa com transtorno mental e sua família ao risco de desassistência, principalmente nos casos que se relacionam ao risco de suicídio. Assim, o Serviço de Emergência Psiquiátrica constitui ferramenta importante para o direcionamento da demanda de cuidados em saúde mental e para a aceitação da doença, no meio social e familiar ${ }^{(4)}$. Neste contexto, ressalta-se a importância de estudos que avaliam as fragilidades e potencialidades das unidades de emergência ${ }^{(5)}$.

De acordo com os desafios impostos pelo novo modelo de atenção em saúde mental, pautado na desinstitucionalização, ressalta-se a importância de investigações sobre a dinâmica assistencial, instituída no ambiente de emergência psiquiátrica, principalmente na perspectiva da enfermagem, uma vez que estes podem contribuir com a diminuição de estigmas e preconceitos, intermediados pelo cuidado humanizado ${ }^{(6)}$. Porquanto, urge a necessidade de conhecer o contexto global onde o cuidado à pessoa acometida pelo transtorno mental se estabelece, de modo a compreender a dinâmica assistencial como um todo e a sua relação com a Rede de Atenção Psicossocial e serviços primários em saúde ${ }^{(7)}$.

Como lacuna do conhecimento ressalta-se a escassez de estudos que avaliem a dinâmica assistência dos novos serviços contemplados na Rede de Atenção Psicossocial (RAPS). Sendo assim, este estudo se justifica na premência de compreender como profissionais de saúde percebem o cuidado ofertado em um serviço de emergência psiquiátrica, inserido no hospital geral. Acredita-se que estes dados podem subsidiar ações que promovam melhor qualidade na assistência em saúde mental.

Com base no exposto, questiona-se: considerando a Reforma Psiquiátrica como modelo de Atenção em Saúde Mental, de que modo a pessoa com transtorno mental é atendida em unidade de Emergência Psiquiátrica? Para responder a essa questão, o presente estudo objetivou compreender as reinvindicações, preocupações e questões construídas por profissionais acerca da dinâmica assistencial de um serviço de emergência psiquiátrica.

\section{CAMINHO METODOLÓGICO}

Estudo de caso, realizado em uma unidade de Emergência Psiquiátrica de um Hospital Geral, no período de fevereiro a junho de 2014, utilizando como referencial teórico-metodológico a Avaliação de Quarta Geração, de abordagem hermenêutica-dialética e de cunho construtivista e responsivo. É hermenêutico porque tem caráter interpretativo, dialético porque propõe o debate das opiniões acerca do objeto em avaliação, construtivista porque possibilita a (re)interpretação dos fatos e, por fim, é responsivo 
porque abarca como aspectos importantes para a avaliação as reinvindicações, preocupações e questões dos grupos de interesse (Stakeholder) para o processo avaliativo ${ }^{(8)}$.

Como grupos de interesse foram elencados 15 profissionais da equipe de saúde da Emergência Psiquiátrica. Como respondente inicial (R1) do círculo de profissionais, foi eleito aquele com maior tempo de atuação no Serviço. Como critérios de inclusão dos profissionais estabeleceram-se: atuar no Serviço há mais de seis meses e prestar cuidados diretos a pessoa com transtorno mental e/ou sua família. Foram excluídos do estudo aqueles profissionais que estavam alocados temporariamente no serviço.

O processo de avaliação utilizando a Quarta Geração foi iniciado por meio do Contato com o campo (reunião com a equipe do Serviço, a fim de explicitar os objetivos e método do estudo); Identificação dos membros que iriam compor o grupo de interesse (os quais seriam indicados pelos profissionais); Desenvolvimento e ampliação das construções conjuntas (reconhecimento das percepções dos membros do grupo de interesse, bem como a análise de documentos pertencentes ao Serviço); Apresentação dos dados para o grupo de interesse (realizada a partir do processo de negociação, que consistiu na organização das construções do grupo, validação das informações e, verificação de consensos). E por fim, Construção dos resultados finais do processo avaliativo (definição dos eixos temáticos, de acordo com os excertos/depoimentos qualificados após negociação).

A coleta de dados foi realizada por meio das técnicas de Observação não Participante (etnografia prévia); Observação Participante e; Entrevista Individual, que foi orientada pelos pressupostos do círculo hermenêutico-dialético.

A Observação não Participante ocorreu no período de fevereiro de 2014, com duração total de 61 horas, com objetivo de reconhecer os aspectos pertinentes à dinâmica assistencial do Serviço.

A etapa correspondente à Observação Participante foi desenvolvida no período de março a junho de 2014, totalizando 152 horas. Foi realizada com o objetivo de analisar, de forma sistematizada, a dinâmica do Serviço, pois a observação participante permite a imersão do pesquisador na dinâmica assistencial, proporcionando uma análise indutiva e compreensiva dos fatos construídos na realidade investigada ${ }^{(9)}$. Vale ressaltar que, nesse período a pesquisadora participou ativamente da dinâmica assistencial estabelecida no Serviço.

Enfatiza-se que o material produzido no processo de observação participante e não participante não foi utilizado, neste artigo, como produto de análise para a elaboração das categorias analíticas, mas sim como apoio para a construção inferencial sobre os depoimentos, bem como foi importante para a aproximação das pesquisadoras do objeto de estudo, pois permitiu uma vivência do contexto real do serviço e experiências que proporcionaram a apuração de atividades desenvolvidas.

O círculo hermenêutico-dialético foi iniciado no mês de maio com o respondente 1 (R1). Ao final da entrevista, foi solicitado ao mesmo, que indicasse outro respondente (R2). Porém, antes da próxima entrevista, os temas, conceitos, ideias, valores, preocupações e questões centrais foram analisados, resultando na construção 1 (C1), que juntamente com as questões iniciais serviu de fonte de informações 
para a entrevista de R2, e assim, sucessivamente.

Como instrumento para direcionar as entrevistas, foi utilizado um roteiro semiestruturado, que inicialmente, continha a seguinte questão norteadora: "Como é o Serviço de Emergência Psiquiátrica onde você atua?"; "Quais as fragilidades e potencialidades desse Serviço?". Como construções que surgiram após a entrevista com o R1, foram incluídas nas entrevistas subsequentes as questões: "Como você percebe o atendimento em saúde no Serviço de Emergência Psiquiátrica" e "Qual é a influência da rede de atenção em saúde mental, na dinâmica de cuidado desse Serviço"? As entrevistas foram realizadas em ambiente reservado, utilizando-se gravador digital.

O conteúdo das entrevistas inicialmente foi analisado por meio do Método Comparativo Constante ${ }^{(8)}$. Para tanto foi realizada a escuta dos depoimentos áudio- gravados, logo após a sua coleta, para identificar as construções de cada respondente e poder apresentar o conteúdo das entrevistas precedentes, nas entrevistas posteriores e desta forma realizar novas formulações acerca das questões identificadas nos depoimentos anteriores.

Com a finalização do círculo hermenêutico dialético, o que ocorreu com a repetição das indicações dos sujeitos, os dados foram transcritos na íntegra, dando-se início à fase de organização e análise dos resultados. Para tanto, foram extraídos elementos dos depoimentos que se combinavam tematicamente e, após isso, os mesmos foram descritos por meio de extratos/excertos, apresentados em eixos temáticos e discutidos na reunião de negociação, com base na Política Nacional de Saúde Mental.

As reuniões de negociação foram previamente agendadas e contaram com a participação da maioria dos entrevistados. Para esse momento, foi produzido um material impresso, contendo a síntese dos dados das entrevistas e entregue a cada membro do grupo. Após, foi realizada explanação dos resultados obtidos nas entrevistas com apresentação oral e uso do Power Point, objetivando a discussão, validação e negociação dos dados como eixo prioritário da avaliação.

Esta pesquisa cumpriu todas as exigências éticas e legais vigentes na Portaria $466 / 12^{(9)}$ e o seu projeto se encontra registrado no Comitê de Ética em Pesquisas com Seres Humanos, da instituição signatária, sob o CAAE: 25786714.0.0000.0104. Todos os participantes assinaram o Termo de Consentimento Livre e Esclarecido (TCLE) em duas vias e, para garantir-lhes o anonimato, os extratos/excertos de seus depoimentos estão identificados pela letra $\mathrm{P}$ (profissional); seguidos de um número indicativo da sequência em que as entrevistas foram realizadas e uma breve caracterização.

\section{RESULTADOS}

\section{Compreendendo a Dinâmica Assistencial do Serviço de Emergência Psiquiátrica}

$\mathrm{Na}$ análise dos depoimentos, que faziam referência a (des)articulação da Emergência Psiquiátrica no hospital geral com os serviços que compõem a Rede de Atenção Psicossocial foi possível identificar potencialidades e fragilidades na edificação de um cuidado resolutivo. 
Durante a observação foi possível identificar que cotidianamente a unidade recebia pessoas com transtorno mental leve, encaminhadas das Unidades Básicas de Saúde, para a solicitação de transcrição de receitas de psicotrópicos. Em média, esse evento acontecia em cinco de cada 10 atendimentos.

O serviço está atendendo mais do que se pretendia e isso acaba prejudicando nossa atuação. Se as pessoas soubessem o tanto de consulta para fazer receita que a gente tem, e o tanto de paciente que a gente coloca para dentro, apenas para medicar e pedir uma vaga na rede, esses gestores iriam valorizar mais o nosso trabalho e dar conta de resolver a falta de serviços na rede. (P3- enfermeira, 37 anos, atua há um ano e seis meses no Serviço)

Os depoimentos também apontaram para a incipiência dos serviços substitutivos. Este fato pode ser considerado como um dos facilitadores para a sobrecarga do Serviço de Emergência.

Quando atendemos uma paciente que não é indicação de internação nós damos os primeiros atendimentos e depois fazemos um encaminhamento para os outros serviços como o CAPS, o que acontece é que sempre o paciente volta na emergência e diz que lá para onde mandamos eles não atendem bem [...]. (P15 - 29 anos, médica, atua há dois anos e um mês no serviço)

Também foi observada a procura da Emergência Psiquiátrica, por pessoas com problemas relacionados ao abuso crônico de substâncias psicoativas, ainda que essas pessoas não apresentassem nenhuma intercorrência psiquiátrica.

O que tumultua aqui são os usuários de drogas, eles vêm para cá muitas vezes para fugir de crimes e vira uma baderna, esses dias mesmo fizeram um motim porque queriam sair de alta e a médica não deixou [...], são poucos os que vem para tratar [...]. (P13 - 33 anos, Auxiliar de enfermagem, atual há quatro anos no serviço)

Outro pormenor que compromete a eficácia da dinâmica assistencial é o constante atendimento de crianças, realizados em decorrência de ordens judiciais para internação compulsória. O constrangimento da equipe frente a este impasse pode ser verificado nos excertos a seguir:

Uma coisa que a gente tem como ponto fraco é o atendimento de crianças encaminhadas por ordem judicial. A nossa dificuldade é imensa porque, a gente não tem estrutura física adequada para esse tipo de atendimento. (P1 - médica, 36 anos, atua há três anos no Serviço)

No que concerne ao quadro de funcionários, o Serviço de Emergência Psiquiátrica em estudo funciona com uma equipe multiprofissional. Todavia, essa equipe é bastante fragilizada, uma vez que, diariamente os funcionários de enfermagem são remanejados para ajudarem em outros setores do hospital. Este enfoque pode ser evidenciado a seguir:

A gente aqui faz milagre na enfermagem, porque veja bem, eu estou aqui na emergência e ao mesmo tempo na clínica, e me diz: "como eu posso fazer enfermagem certo em dois lugares ao mesmo tempo?" [...]. (P12 - 37 anos, enfermeira, atua há quatro anos no serviço)

Mesmo com o quadro de funcionários reduzido, foi verificado, durante o período de observação que, mesmo com um número inadequado de profissionais, a equipe multidisciplinar se diligencia em ofertar um 
cuidado acolhedor.

A desarticulação da Rede de Atenção Psicossocial como entrave para a satisfação sobre a Assistência na Emergência Psiquiátrica

Com relação à dinâmica assistencial estabelecida na Emergência Psiquiátrica, o que mais chamou a atenção é a desarticulação da Rede de Atenção Psicossocial com o Serviço. Esta proposição pode ser evidenciada nas falas a seguir:

O pessoal dos outros serviços manda todos os pacientes psiquiátricos para a Emergência porque, é aquela coisa básica de não ter serviço que cuide disso na Rede. Com isso a gente não consegue dar conta de cuidar de todos com resolutividade e isso, faz com a gente fique perturbado. (P1 - médica, 36 anos, atua há três anos no Serviço)

A admissão frequente de crianças na Emergência é um fator que também agrega insatisfação no trabalho profissional, e este aspecto, é agravado pelo longo tempo de permanência desses casos no Serviço, associado à fragilidade estrutural para o atendimento infanto-juvenil.

O que complica muito a rotina de cuidado aqui é a internação de crianças porque a Rede não tem estrutura para isso. Então, o jeito é internar na Emergência. [...] Mas é preciso considerar que aqui não é o lugar adequado, é um lugar fechado, só um corredor, cheio de adultos. [...] acaba atrapalhando a nossa forma de cuidar, deixando a gente triste em ver essas crianças trancadas aqui. (P8 - médico, 31 anos, atua há três anos no Serviço)

A desarticulação da RAPS com o Serviço de Emergência Psiquiátrica foi apontada nos excertos anteriores como a precursora de grande insatisfação entre os profissionais, principalmente em decorrência da desassistência nas unidades de saúde que compõem a RAPS, associada à sobrecarga de cuidados na unidade de Emergência.

\section{DISCUSSÃO}

A precariedade da Rede de cuidados em saúde mental acarreta sobrecarga de atendimentos nas unidades de emergência ${ }^{(10-11)}$. Outro aspecto relevante é que, mesmo o Serviço de Emergência Psiquiátrica não ter como prioridade a transcrição de receitas, o mesmo cumpre com seu papel de articulador do cuidado em saúde mental quando, "colocam para dentro", pessoas para serem medicadas, denotando com isso o comprometimento com a resolutividade dos casos e consequentemente o vínculo com o usuário.

$\mathrm{Na}$ concepção dos profissionais os usuários durante o atendimento relatam que a assistência na Emergência Psiquiátrica é melhor e por isto eles referem procurar este serviço do que fazer acompanhamento em outros pontos da rede de atenção psicossocial.

Sobre os serviços que compõem a Rede Psicossocial, estudo realizado no oeste Paulista, com o objetivo de identificar as ações desenvolvidas na Estratégia Saúde da Família junto às pessoas com transtorno mental e sua família, concluiu que as pessoas que necessitam de cuidado em saúde mental na atenção primária sofrem com a desassistência ${ }^{(12)}$ denotando assim, a emergente necessidade de ações voltadas à melhor 
prática de cuidado em saúde mental dentro da comunidade.

A fragilidade na RAPS também se faz presente no contexto do tratamento para dependência química. Nesta pesquisa a procura pela unidade de atendimento em Emergência Psiquiátrica, por pessoas com necessidade de cuidados relacionados à dependência química acontece pela incipiência de serviços públicos que prestam cuidados específicos a esta população, fazendo do Serviço de Emergência Psiquiátrica a "Porta de Entrada" para o atendimento na Rede de cuidados em Saúde Mental, que pelo contexto descrito, apresenta-se frágil no que concerne à oferta de vagas.

A fragilidade no atendimento para dependência química é também realidade de outras regiões. Por exemplo, estudo que fez uma análise retrospectiva da demanda de atendimento emergencial na região central do Rio Grande do Sul identificou um número significativo de atendimentos, determinados principalmente pelo uso/abuso de substâncias psicoativas, com maior prevalência do uso de álcool ${ }^{(13)}$.

Outra problemática apontada neste estudo refere-se ao sentimento de indignação nas falas de P1 e P7 ao mencionarem que crianças ficam "morando" em um Serviço de Emergência, sem que este tenha estrutura adequada para este tipo de atendimento. Este aspecto denota a gravidade da situação de cuidados de saúde mental para crianças. O longo tempo de permanência, associado a falta de estrutura adequada para a assistência infantil faz emergir a necessidade de discussões acerca dos direitos da criança, como forma de salvaguardar sua integridade psíquica enquanto Ser em crescimento e formação.

Estudos apontam a desassistência a crianças com transtornos mentais, ao evidenciar aumento na demanda de ocorrências nas unidades de saúde, que, na maioria dos casos apresentam-se fragilizadas para assistir crianças e adolescentes ${ }^{(14-15)}$.

É preciso então, que os gestores se atentem às necessidades para o atendimento ao cuidado em saúde mental infantil, estabelecidas por normas éticas vigentes nas políticas públicas de saúde, que exige novas formas de estruturar e transitar entre os Serviços, com vistas a consolidar a atenção acolhedora, de forma a não reproduzir o modelo hospitalocêntrico e segregativo, preconizado nas instituições psiquiátricas asilares, mantendo assim os princípios da bioética como precursores do cuidado humanizado em saúde ${ }^{(16)}$.

Em alguns momentos, observa-se que a unidade de emergência fica fragilizada no que concerne ao cuidado especializado em saúde mental, levando a diminuição da qualidade das ações de saúde, associada a sobrecarga dos funcionários.

De acordo com as fragilidades e potencialidades apontadas pelos participantes, é possível afirmar que mesmo com muitas dificuldades na construção de uma dinâmica assistencial qualificada, o Serviço de Emergência Psiquiátrica, inserido no hospital geral tenta se fortalecer atuando como local de cuidado humanizado, edificando ações de saúde de acordo com as possibilidades que o meio oferece.

No entanto, é possível asseverar que a escassez de serviços que compreendem a RAPS é um complicador para a qualidade da atenção na Emergência ${ }^{(11)}$. Este fato é comprovado quando o profissional 1 exterioriza não conseguir proporcionar ações de saúde mais resolutivas, sentindo-se assim insatisfeito com a qualidade do cuidado ofertado, bem como com a decepção do familiar em não ter outras oportunidades 
de cuidado.

A elevada demanda de atendimentos psiquiátricos em um único serviço pode fragilizar a qualidade do cuidado ofertado, uma vez que são realizadas ações de saúde pontuais, fazendo emergir a necessidade de maior intervenção, por parte das Políticas Públicas de saúde, na construção de serviços que abarquem a doença mental como objeto de cuidado para a humanização da assistência ${ }^{(17)}$.

Os depoimentos também evidenciam o descompasso existente em se realizar tratamento de crianças com adultos, em uma unidade de Psiquiatria. No contexto do atendimento infantil em saúde mental as unidades de Emergência Psiquiátrica têm como dever identificar o quadro clínico inicial, os riscos decorrentes da presença do transtorno mental, os fatores que levam à agudização ou agravamento do quadro clínico, bem como a presença de suporte familiar, social e de serviços de saúde extra-hospitalar para continuidade do tratamento destas crianças e suas famílias ${ }^{(15)}$.

A preocupação da equipe de saúde frente à internação judicial de menores também foi evidenciada nos momentos em que os profissionais tentavam convencer a gestão do hospital, sobre a fragilidade da estrutura física e de recursos humanos para abarcar esse tipo de atendimento. Contudo, não havia sucesso nestes momentos de negociação, fazendo com que a equipe se responsabilizasse pelos cuidados disciplinares de jovens internados na Emergência.

No contexto do cuidado psiquiátrico, a nova forma de fazer saúde mental exige a construção de serviços de saúde que possibilitem a consolidação de ações que favoreçam o fluxo da dinâmica de cuidado na RAPS, tanto para adultos quanto para crianças ${ }^{(17)}$, objetivando que o cuidado em saúde seja um objeto de humanização e de transformação do modelo excludente ${ }^{(18)}$. Sendo assim, é importante que se amplie a oferta de vagas extra-hospitalares para a continuidade do tratamento iniciado nas unidades de Emergência.

Em suma, é possível inferir que a dinâmica assistencial construída na unidade de Emergência luta constantemente para se articular com outros serviços da RAPS. Contudo, o desgaste físico e emocional, associados à insatisfação dos profissionais, parecem ser complicadores da construção de ações de saúde mais resolutivas.

\section{CONSIDERAÇÕES FINAIS}

Por meio deste estudo foi possível inferir que a dinâmica assistencial estabelecida na Emergência Psiquiátrica tenta direcionar o fluxo de usuários para o tratamento adequado, uma vez que o Serviço luta constantemente para relacionar-se com a Rede de Atenção Psicossocial, com vistas a melhorar a articulação entre as unidades de cuidado. Considerando todos os desafios vivenciados, acredita-se que a dinâmica assistencial desse Serviço extrapola o mero caráter de unidade de estabilização de pacientes com quadros de agudização do transtorno mental.

Também foi possível constatar que os profissionais apresentam insatisfação frente aos entraves impostos pela desarticulação da Rede com a Emergência Psiquiátrica, sendo este o maior desafio para a continuidade do cuidado em saúde mental, bem como para a consolidação da Política Nacional de Saúde 
Mental, bem como da Política Nacional de Humanização.

De acordo com as fragilidades descritas, é possível apreender que a qualidade do cuidado ofertado no Serviço de Emergência Psiquiátrica é comprometida pelo descompasso existente entre aspectos específicos da dinâmica assistencial, como: espaço físico inadequado para atendimento de crianças, elevado número de atendimentos, desfalque no quantitativo de profissionais e, enfermeiro sobrecarregado com atividades.

Como potencialidade, no que concerne à utilização do método de Avaliação de Quarta Geração, é possível afirmar que este referencial teórico-metodológico, que abarca a pesquisa construtivista, com enfoque formativo e responsivo, faz emergir o empoderamento dos grupos de interesse como protagonistas das transformações necessárias ao aprimoramento do Serviço, uma vez que, por meio da negociação, prevista no método de pesquisa, foi possível estabelecer propostas de adequação dos aspectos a serem reestruturados.

Com relação aos atendimentos de crianças foi estabelecida a construção de um relatório, por toda a equipe de profissionais, reivindicando a execução, em médio prazo, de um projeto já existente, de construção de uma unidade de atendimento infantil a fim de extinguir o atendimento de crianças junto com adultos no Serviço de Emergência Psiquiátrica.

Como limitação deste estudo aponta-se o fato de os dados refletirem as informações obtidas em um determinado momento e em local específico. Desse modo, os dados estão sujeitos à influências temporais e socioculturais. Sendo assim, o processo de pesquisa sistematizado nesse trabalho, não pretende fazer generalizações, para além dos limites do local de estudo e, em nenhum momento, tem a pretensão de esgotar a temática.

Estabelecer planos de melhoria das unidades de saúde ainda é um desafio a ser superado. Neste contexto, o estudo de avaliação de Quarta Geração, possibilitou trazer à tona um tema atual e de grande relevância para a saúde pública, que é a desinstitucionalização da pessoa com transtorno mental de forma a garantir um atendimento em saúde resolutivo e com qualidade.

\section{REFERÊNCIAS}

1. Trapé TL, Campos RO. The mental health care model in Brazil: analyses of the funding, governance processes, and mechanisms of assessment. Rev Saude Publica [Internet]. 2017 [acesso em: 31 dez. 2017];51:19. Disponível em: http://doi.org/10.1590/s1518-8787.2017051006059.

2. Siqueira-Silva R, Nunes JA, Moraes M. Portugal e Brasil no cenário da saúde mental. Fractal: Rev Psicol [Internet]. 2013 [acesso em: 31 dez. 2017];25(3):475-96. Disponível em: http://doi.org/10.1590/S1984-02922013000300005. 3. Willrich JQ, Kantorski LP, Chiavagatti FG, Cortes JM, Antonacci MH. Os sentidos construídos na atenção à crise no território: o Centro de Atenção Psicossocial como protagonista. Rev esc enferm USP [Internet]. 2013 [acesso em: 31 dez. 2017];47(3):657-63. Disponível em: http://doi.org/10.1590/50080-623420130000300019.

4. Horwitz AG, Czyz EK, King CA. Predicting Future Suicide Attempts Among Adolescent and Emerging Adult Psychiatric Emergency Patients. J Clin Child Adolesc Psychol [Internet]. 2015 [acesso em: 31 dez. 2017];44(5):751-61. Disponível em: http://doi.org/10.1080/15374416.2014.910789. 
5. Silva PL, Paiva L, Faria VB, OhI RIB, Chavaglia SRR. Triage in an adult emergency service: patient satisfaction. Rev esc enferm USP [Internet]. 2016 [acesso em: 31 dez. 2017];50(3):427-33. Disponível em: http://doi.org/10.1590/S0080623420160000400008.

6. Buriola AA, Kantorski LP, Sales CA, Matsuda LM. Nursing practice at a psychiatric emergency service: evaluation using fourth generation assessment. Texto contexto - enferm. [Internet]. 2016 [acesso em: $31 \mathrm{dez}$.

2017];25(1):e4540014. Disponível em: http://doi.org/10.1590/0104-070720160004540014.

7. Lund C, Tomlinson M, Patel V. Integration of mental health into primary care in low- and middle-income countries: the PRIME mental healthcare plans. Br J Psychiatry [Internet]. 2016 [acesso em: 31 dez. 2017];208(s56):s1-3.

Disponível em: http://doi.org/10.1192/bjp.bp.114.153668.

8. Guba EG, Lincoln YS. Avaliação de quarta geração. Campinas: Unicamp, 2011.

9. Resolução No 466 do Conselho Nacional de Saúde, de 12 de dezembro de 2012 (BR) [Internet]. Aprova as diretrizes e normas regulamentadoras de pesquisas envolvendo seres humanos. Diário Oficial da União. 12 dez. 2012 [acesso em: 31 dez. 2017]. Disponível em: http://bvsms.saude.gov.br/bvs/saudelegis/cns/2013/res0466_12_12_2012.html. 10. Jones AL, Cochran SD, Leibowitz A, Wells KB, Kominski G, Mays VM. Usual Primary Care Provider Characteristics of a Patient-Centered Medical Home and Mental Health Service Use. J Gen Intern Med [Internet]. 2015 [acesso em: 31 dez. 2017];30(12):1828-36. Disponível em: http://doi.org/10.1007/s11606-015-3417-0.

11. Molina-López A, Cruz-Islas JB, Palma-Cortés M, Guizar-Sánchez DP, Garfias-Rau CY, Ontiveros-Uribe MP, et al. Validity and reliability of a novel Color-Risk Psychiatric Triage in a psychiatric emergency department. BMC Psychiatry [Internet]. 2016 [acesso em: 31 dez. 2017];16(1):30. Disponível em: http://doi.org/10.1186/s12888-016-0727-7. 12. Fioramonte A, Bressan BF, Silva EM, Nascimento GL, Buriola AA. Cuidado à pessoa com transtorno mental e sua família: atuação do enfermeiro na ESF. Cienc Cuid Saude [Internet]. 2013 [acesso em: 31 dez. 2017];12(2):315-22. Disponível em: http://doi.org/10.4025/cienccuidsaude.v12i2.20362.

13. Calegaro VC, Bertuol Filho A, Lima JARF, Andrade GT, Dahmer CM, Lunelli LB, et al. Padrão dos atendimentos em uma emergência psiquiátrica de referência para a Região Central do Rio Grande do Sul. Revista da AMRIGS [Internet]. 2016 [acesso em: 31 dez. 2017];60(3):185-90. Disponível em: http://www.amrigs.org.br/revista/60-

03/padrao_atendimento.PDF.

14. Kantorski LP, Nunes CK, Sperb LCSO, Pavani FM, Jardim VMR, Coimbra VCC. The intersectoriality in the psychosocial attention of children and adolescent. Revista de Pesquisa: Cuidado é Fundamental Online [Internet]. 2014 [acesso em: 31 dez. 2017];6(2):651-62. Disponível em:

http://www.seer.unirio.br/index.php/cuidadofundamental/article/view/3210.

15. Lunsky Y, Paquette-Smith M, Weiss JA, Lee J. Predictors of emergency service use in adolescents and adults with autism spectrum disorder living with family. Emerg Med J [Internet]. 2015 [acesso em: 31 dez. 2017];32(10):787-92. Disponível em: http://doi.org/10.1136/emermed-2014-204015.

16. Williams AR. Opportunities in Reform: Bioethics and Mental Health Ethics. Bioethics [Internet]. 2016 [acesso em: 31 dez. 2017];30(4):221-6. Disponível em: http://doi.org/10.1111/bioe.12210.

17. Antonacci MH, Kantorski LP, Willrich JQ, Argiles CTL, Coimbra VCC, Bielemann VLM. Estrutura e fluxo da rede de saúde como possibilidade de mudança nos serviços de atenção psicossocial. Rev esc enferm USP [Internet]. 2013 [acesso em: 31 dez. 2017];47(4):891-8. Disponível em: http://doi.org/10.1590/S0080-623420130000400017. 18. Oliveira EB, Medonça JLS. Family member with chemical dependency and consequent burden suffered by the family: descriptive research. Online Brazilian J Nurs [Internet]. 2012 [acesso em: 31 dez. 2017];11(1):14-24. Disponível em: http://doi.org/10.5935/1676-4285.20120003. 